Importancia de la ética en la Educación Superior

Número Publicado el 05 de abril de 2018

\title{
Importancia de la ética en la Educación Superior
}

\author{
Importance of ethics in higher education
}

\section{Importância da ética no ensino superior}

\author{
Cristian G. Méndez-Medrano ${ }^{\mathrm{I}}$ \\ cristian.mendezm@ug.edu.ec \\ Mario W. Torres-Gangotena ${ }^{\text {II }}$ \\ mario.torresg@ug.edu.ec \\ Segundo B. Camatón-Arizábal III \\ segundo.camatona@ug.edu.ec
}

Recibido: de noviembre de 2017 * Corregido: 18 de febrero de 2018 * Aceptado: 10 de marzo de 2018

1. Magister en Educación Con Mención Enseñanza de la Matemática, Ingeniero Comercial, Universidad de Guayaquil, Guayaquil, Ecuador

II. Magister en Educación con Mención Enseñanza de la Matemática, Licenciado en Ciencias de la Educación Mención Educación Básica, Universidad de Guayaquil, Guayaquil, Ecuador.

III. Magister en Educación con Mención Enseñanza de la Matemática, Doctor En Ciencias de la Educación Especialización Físico Matemáticas, Universidad de Guayaquil, Guayaquil, Ecuador. 


\section{Resumen}

Este artículo está basado en mostrar los principales desarrollos y problemas en la enseñanza de la ética a estudiantes de pregrado. La enseñanza de la ética ha estado única e inseparablemente conectada con los asuntos más importantes de la educación superior moderna, temas que involucran el currículum, la institucionalización, la profesionalización, la epistemología. Desde esta perspectiva más amplia, se ha intentado comprender el lugar y los problemas de la enseñanza de la ética, no solo con respecto a los cursos de ética como tales, sino también en un plan de estudios más amplio. A pesar de que la preocupación por la enseñanza de la ética ha sido embaucada una y otra vez o incluso ha desaparecido en algún momento de la educación superior, sin excepción ha vuelto y casi de inmediato en la mayoría de los pensum de estudio en las universidades. Las grandes potencias hoy en día llevan años incluyendo esta práctica en sus estudios lo que les ha permitido desarrollar a sus ciudadanos de una manera tal que han impulsado a su país en los sectores más importantes del ámbito económico, social y cultural. Copiar esta metodología en nuestra región nos llevaría a equipararnos con esas naciones en los altos niveles mundiales de educación y producción.

Palabras clave: ética; educación; metodología; universidades.

\section{Abstract}

This article is an attempt to see the main developments and problems in the teaching of ethics to undergraduate students. The teaching of ethics has been uniquely and inseparably connected with the most important issues of modern higher education, issues that involve the curriculum, institutionalization, professionalization, epistemology. From this broader perspective, an attempt has been made to understand the place and problems of teaching ethics, not only with respect to ethics courses as such, but also in the broader curriculum. Even though the concern for the teaching of ethics has been duped again and again or even disappeared at some point in higher education, without exception it has returned and almost immediately in most of the study pensum in the universities. The great powers nowadays have been including this practice in their universities for years, which have allowed them to develop their citizens in such a way that they have promoted their country in the most important economic, social and cultural sectors. Copying this methodology in our region would lead us to equate ourselves with those nations in the high levels of education and production worldwide.

Keywords: ethics; education; methodology; universities. 


\section{Resumo}

Este artigo baseia-se em mostrar os principais desenvolvimentos e problemas no ensino da ética para alunos de graduação. $\mathrm{O}$ ensino da ética tem sido singular e inseparavelmente ligado às questões mais importantes do ensino superior moderno, questões que envolvem o currículo, a institucionalização, a profissionalização, a epistemologia. A partir dessa perspectiva mais ampla, tentou-se entender o lugar e os problemas do ensino de ética, não apenas no que diz respeito aos cursos de ética como tal, mas também em um currículo mais amplo. Mesmo que a preocupação com o ensino da ética tenha sido repetidamente enganada ou até mesmo desaparecido em algum momento do ensino superior, sem exceção, ela retornou e quase imediatamente na maioria dos estudos pensum nas universidades. As grandes potências de hoje têm incluído essa prática em seus estudos há anos, o que lhes permitiu desenvolver seus cidadãos de tal forma que eles promoveram seu país nos setores econômicos, sociais e culturais mais importantes. Copiar essa metodologia em nossa região nos levaria a nos equiparar às nações nos altos níveis de educação e produção em todo o mundo.

Palavras chave: ética; educação; metodologia; universidades.

\section{Introducción}

En nuestra era actual, la ética tiene un lugar importante en todas las áreas de la vida. La ética también es parte importante en la educación, porque la educación es un proceso fundamental de la vida humana. Actualmente tenemos el alcance fácilmente a todo el conocimiento a través de la tecnología. En ocasiones, en la educación, el uso de la tecnología revela cierta falta de ética, problemas como el plagio de trabajos de investigación podría ser uno de ellos. Para comprender la importancia de la ética, esta debe colocarse como un curso en el sistema educacional. Antes de discutir este tema, es necesario definir qué es la ética y qué es la educación.

La ética es la rama más importante y funcional de la filosofía en la actualidad. En general, la ética es moral filosófica. El término ética se deriva del término griego Ethos que significa costumbre, carácter. Está relacionado con nuestros valores y virtudes. Por lo tanto, nuestras acciones y nuestras experiencias en la vida cotidiana son temas de ética.

La ética es la ciencia del deber ser que provee las pautas para obrar honestamente. O sea que la ética es una ciencia normativa. Pero no sólo una ciencia de deberes: es también la ciencia de la perfección moral mediante la adquisición o aprendizaje de las virtudes o hábitos positivos adoptados por la 
persona para obrar rectamente en uno de los sectores del obrar. Por ejemplo: el patriotismo, el civismo, el altruismo, la amistad, la lealtad, la laboriosidad, la prudencia, la valentía, etc. (Posada, 1997).

Una preocupación por la instrucción ética y la formación de los estudiantes siempre ha sido parte de la educación superior moderna. Sin embargo, esa preocupación de ninguna manera ha sido uniforme ni está libre de controversia. A mediados del siglo XX, las instrucciones sobre ética se habían limitado, en general, casi exclusivamente a los departamentos de filosofía y religión. Los esfuerzos para introducir la enseñanza de la ética en las escuelas profesionales y en otras partes de la universidad a menudo se encontraron con indiferencia u hostilidad total. La última década ha visto un notable resurgimiento del interés en la enseñanza de la ética, especialmente en los niveles de la escuela profesional.

¿Qué pasaría si la educación superior tomara un papel de liderazgo, como lo hizo en la carrera espacial y la guerra contra el cáncer, en la preparación estudiantes y proporcionando la información y el conocimiento para lograr una sociedad éticamente responsable? La educación de todos los profesionales reflejaría un nuevo enfoque de aprendizaje y práctica. La universidad o colegio funcionaría como un sistema totalmente integrado comunidad que modela la sostenibilidad social.

La ética como disciplina insta a las universidades a comprometerse con una docencia que consolide principios éticos y deontológicos propios de los diferentes marcos profesionales. Por otra parte, las instituciones universitarias asumen la obligación de otorgar una educación que enaltezca valores personales y sociales. Se necesita, entonces, un desarrollo docente que evidencie principios valóricos fundamentales, para que sirvan de modelo y orienten la formación (Arratia Figueroa, 2008).

Se examinan los principales problemas y cuestiones de ética en la educación superior. Se consideran la función y el estado actual de la ética profesional, junto con códigos de ética específicos, De especial interés es si los estándares están universalizados y si existen similitudes o diferencias entre códigos y consistencia lógica. También se consideran: la justificación de la ética profesional, la libertad académica, el uso ético de pruebas y exámenes, la libertad de los estudiantes para aprender, la investigación con sujetos humanos, la financiación de proyectos de investigación, los conflictos de intereses y la deshonestidad en la investigación. 
La formación ética del profesional no debiera reducirse a la internalización de la eticidad profesional existente, sino tendría que contribuir a la configuración de una personalidad capaz de criticar los valores existentes... sobre todo, actuar reflexiva y autocríticamente para modificar sus esquemas y prácticas teniendo como horizonte la dignidad de todos y todas (López Zavala, 2013).

\section{Metodología}

Esta investigación se enfoca en el estudio y análisis de la ética y valores en la educación superior, con el propósito de crear mejores profesionales en los distintos ámbitos de la vida cotidiana.

Se trata de una revisión bibliográfica vigente de textos, documentos e informes disponibles en el web considerando que aquella herencia de la globalización nos permite acceder a mayor y mejor información a través de las herramientas tecnológicas. El motor de búsqueda han sido herramientas académicas de la web que direccionan específicamente a archivos con validez y reconocimiento.

\section{Resultados}

Una parte importante del trabajo de la inserción de la ética como tema fundamental en la educación superior fue la recopilación de información sobre actividades en esta área a nivel universitario y de escuela profesional. Hay una cantidad significativa de actividad en el área de la instrucción ética. De hecho, el número de personas y escuelas involucradas en la educación ética es tan grande, que cualquier descripción de actividades y programas será por necesidad algo incompleta y desactualizada. La instrucción ética se está desarrollando rápidamente en muchas escuelas, y constantemente se agregan nuevos programas y cursos al plan de estudios. Al enfrentar la enorme tarea de describir la amplia gama de actividades que existe actualmente en la enseñanza de la ética, durante la investigación se aprovechó varias estrategias para muestrear los datos disponibles.

El estudio de la ética en la educación superior respecto a la conducta de los estudiantes, profesores y científicos, es un ámbito de análisis relevante en las ciencias sociales, tanto desde la perspectiva del ejercicio profesional como a nivel institucional de cada universidad (Hirsch, 2012). 
Fig. 1. Interacción de la ética en la Educación Superior

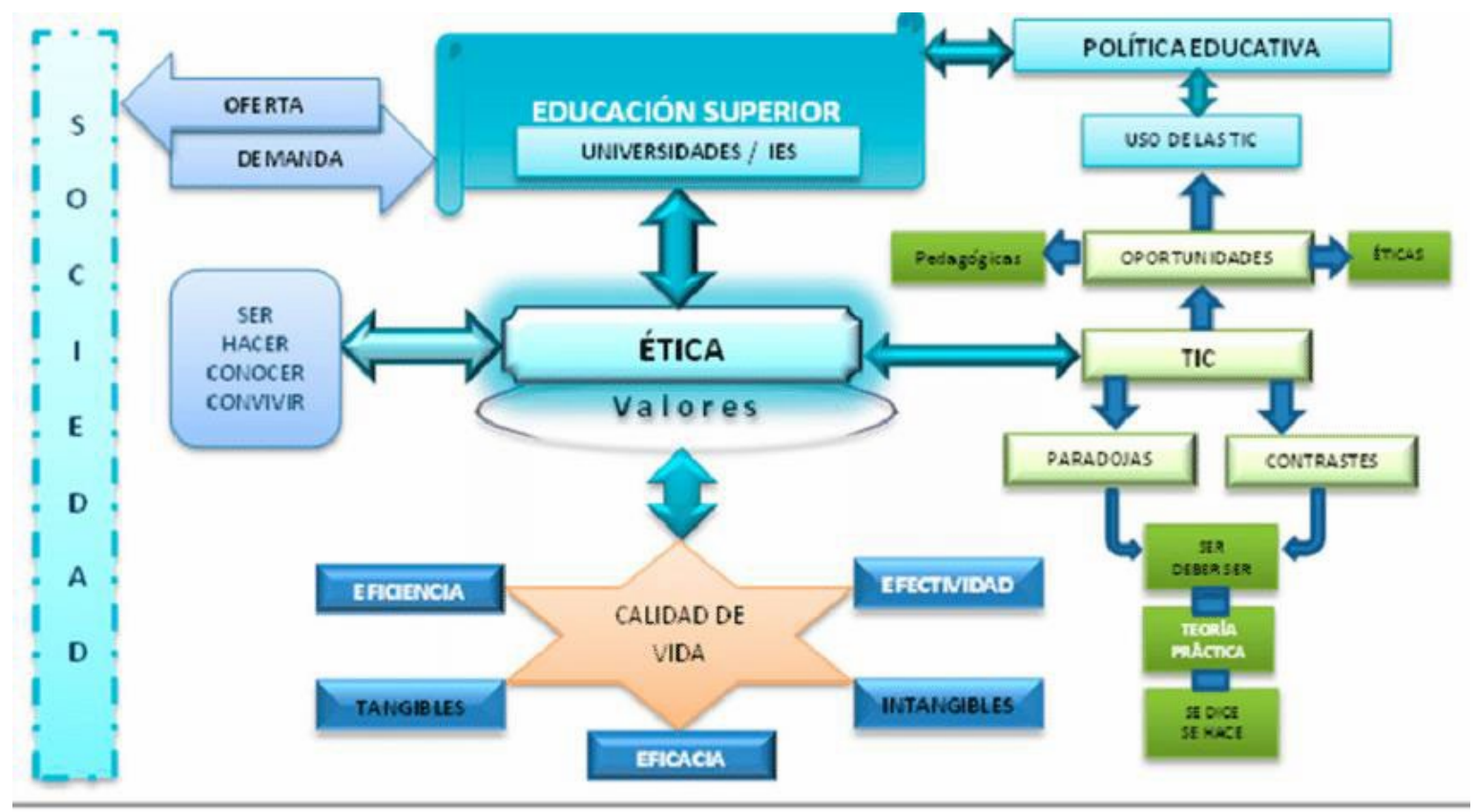

Fuente: García (2013)

La perspectiva organizacional del comportamiento ético de las instituciones universitarias abre un espacio de análisis interesante para conectarlo con el concepto de Responsabilidad Social Universitaria (RSU), entendida desde una perspectiva ética como un compromiso institucional permanente del quehacer universitario con las necesidades y problemáticas de la sociedad en la cual la universidad se inserta, mediante una relación transparente, fluida y permanente con el tejido social y sus partes interesadas. Así, hoy las universidades, desde una perspectiva organizacional, poseen un importante rol ético que deben cumplir en las sociedades modernas de las que forman parte, situación plasmada en la referida Conferencia Mundial de Educación Superior (París, 1998), organizada 29 por la Unesco reclamando a las universidades para que asumieran una mayor Responsabilidad Social en el contexto de su papel en la sociedad del conocimiento, en especial en la producción de conocimiento científico demandado socialmente (De la Calle et al., 2007; Gaete, 2011).

Una forma particular de ética profesional es específica del rol del docente entre otros educadores, ya sean padres, "ministros de religión, entrenadores deportivos o líderes de recreación". Los docentes a veces toman decisiones basadas en una cierta ética profesional intuitiva y personal. Pero no necesariamente lo comparten con sus colegas. El propósito de definir la ética profesional de la enseñanza es nombrar y aclarar una ética común para todos los miembros de la profesión docente. Podrán afirmarlo y testificar abiertamente en sus intervenciones. Para aclarar esto, utilizaremos las 
características de las ocupaciones presentadas en el primer capítulo y las relacionaremos con el trabajo de los docentes para identificar las consecuencias éticas.

Hoy nos enfrentamos al crecimiento vertiginoso del volumen de información y la transmisión de la cultura, en un medio en que hay crisis en las instituciones políticas y sociales (las escuelas y universidades, las empresas, el poder legislativo, políticos, gobierno...); en que los recursos son escasos, se estanca el crecimiento económico; aumenta las protestas sociales y dominan los intereses corporativos. Concomitantemente hay un deterioro del nivel ético profesional en todos los niveles de la sociedad. La masificación de la universidad puede ser señal de que la persona es valorada y no se discrimina por sus recursos económicos, lo cual es positivo. En cambio, al crecer el número de estudiantes, falla el contacto entre profesores y alumnos, así como de los profesores entre sí. La formación se centra más en la especialización, y se olvida el estudio de los códigos de conducta por los que debe regirse toda profesión liberal. La ética es muy importante, porque los valores morales que se aprenden en las aulas son los que influyen en su comportamiento profesional. La educación superior supone el desarrollo total del individuo, en que a la formación profesional debe sumarse la formación humanística. Así, una persona está bien educada si es capaz de analizar críticamente su sociedad, si está formada para tomar decisiones éticas, y por supuesto, si tiene conocimientos especializados en que se va a apoyar su futura carrera profesional (Barros, 2015).

Peor aún es la escasa atención que nuestros jóvenes y los mismos catedráticos ponen en lo realmente importante: la formación de seres humanos de alto desempeño ético y en su lugar se ufanan con los logros de algunos "genios" -así llamamos a aquellos que desarrollan la inteligencia lógico matemáticacon elevado coeficiente intelectual pero que se vuelven incapaces para interactuar y dirigir una empresa generadora de bienestar social. En cambio terminan sin trabajo o "atrapados" al servicio de la gente dueña del capital Pero dentro del vaso medio vacío hay una esperanza: Al fin el sistema educativo toma el papel que le corresponde y permite la incorporación de materias con contenidos éticos y humanísticos en los programas de educación superior; lo que delega al profesor una gran responsabilidad, pero a la vez lo dota de una herramienta básica para lograr su objetivo: profesionistas comprometidos con la sociedad y su entorno. Si bien es cierto que este primer paso no lo es todo, también lo es que nos genera expectativas positivas para usar en el futuro un modelo educativo en el que se privilegie el hacer por el conocer, el ser por el tener y el comprender por el saber (Correa, 2004). 
Sin embargo, los cambios que se están produciendo en la educación superior en muchas partes del mundo también están ayudando a dar forma a una nueva identidad profesional para los profesores universitarios. La expansión de los cursos vocacionales y profesionales en las universidades significa que un doctorado ya no es el punto de partida normal para todas las carreras académicas. Los docentes en áreas tales como negocios y administración, trabajo social, educación y enfermería tienen más probabilidades de tener experiencia profesional o vocacional en lugar de un doctorado. De hecho, cada vez son más populares los doctorados profesionales de orientación práctica en administración, educación y ciencias de la salud, tomados después, en lugar de antes, de la experiencia profesional. Mientras que algunos miembros del personal con formación profesional y profesional pueden desear perseguir objetivos de investigación, otros pueden experimentar la presión de realizar investigaciones y publicar como alienantes e inútiles para sus ambiciones profesionales.

Además, los profesores universitarios de los campos profesionales traen consigo valores de sus diversas profesiones. Entre estos valores se encuentra un compromiso con las necesidades de los "clientes" o "clientes" y una menor incomodidad al aplicar este lenguaje a sus propios alumnos. A su vez, esta preocupación por satisfacer las necesidades del 'usuario final' hace que muchos profesores de campos profesionales se adapten a las necesidades del estudiante como destinatario directo de una educación universitaria, mientras está preparado para reconocer las expectativas de los interesados, como los empleadores y organizaciones profesionales.

\section{Conclusión}

Debido a que los colegios y universidades crean y diseminan el conocimiento, y debido al poder que el conocimiento crea, las instituciones de educación superior poseen una responsabilidad moral con la sociedad. El papel de los académicos es crucial debido a su poder para definir la realidad y ejercer control sobre la sociedad en general y los estudiantes en particular. A menudo existen problemas éticos debido a las necesidades competitivas de los diversos roles asumidos por el académico, que pueden concebirse en cuatro fases: enseñanza, descubrimiento, aplicación e integración.

Para ayudar a lidiar con estos problemas, la institución misma debe asumir un papel de liderazgo al formular adecuadamente las declaraciones de la misión basadas en prácticas y preocupaciones éticas; fomentando la colaboración entre todos los profesores, administradores, personal y estudiantes para trabajar con los valores necesarios para la efectividad institucional y la integridad general; y al emplear el uso de modelos de toma de decisiones éticas. El ambiente académico debe fomentar la importancia de la dignidad humana, la nutrición del crecimiento y el logro, y el respeto por los demás. Tal ambiente 
es una comunidad de aprendizaje: una comunidad que reúne los temas de liderazgo, facultad y estudiantes. El liderazgo es esencial para la sensibilidad de una institución a los valores en la educación superior. La comunidad de aprendizaje también puede sacar lo mejor de la facultad y resolver varias de las tensiones que enfrentan los docentes en sus carreras, especialmente los conflictos entre la investigación y la enseñanza. Finalmente, la comunidad de aprendizaje proporciona dirección a los estudiantes y ancla su experiencia universitaria en la vida intelectual.

La ética es la base de nuestra relación humana con nosotros mismos y el mundo que nos rodea. El propósito y el papel de la ética siempre ha sido la preservación del ser humano como persona, la dignidad humana y las condiciones para llevando una buena vida. El tiempo de hoy, la cultura de hoy en día en la que vivimos se caracteriza principalmente por el pluralismo con el que tenemos que lidiar, con las crisis y la agitación que estamos experimentando, con la interconectividad creciente de la mundo (globalización) y la dependencia de los demás, y la "relativización" de los valores, que es principalmente una expresión de la disminución de la confianza en la sociedad y la pérdida de certeza sobre las respuestas a los principios preguntas de nuestra existencia La ética protege y nutre a la humanidad de nuestra existencia, tanto en nosotros mismos como en otros, y la práctica del diálogo es esencial. Siempre vivimos en relación con los demás, es decir, en una relación de mutuo dar y recibir, por lo tanto, el reconocimiento de nuestra dependencia de los demás y el cuidado de los demás es esencial (Emil, 2012).

\section{Referencias Bibliográficas}

Barros, G. G. (2015). Educación superior y formación ética.

Correa, A. (2004). Ética social en la educación superior. [en línea], Disponible en: https://www.gestiopolis.com/etica-social-educacion-superior/

Emil, D. (2012). Educación Moral.

Arratia Figueroa, A. (2008). Ética, solidaridad y "aprendizaje servicio" en la educación superior. Acta Bioética, 14 (1), 61-67.

López Zavala, R. (2013). Ética profesional en la educación superior. Perfiles Educativos, XXXV (142), 2-5.

Hirshc, D; Gufberg, E; Ogur, B; Cohen, P; Krupat, E; Cox, M; Pelletier, S y Bor, D. (2012) Educational Outcomes of the Harvard Medical School-Cambridge Integrated Clerkship: A Way Forward for Medical Education. Academic Medicine, 87 (5), 643-650.

Posada O. (1997) Formación ética en la educación. P. 34. 